\title{
Structure variation and evolution in microphase-separated grafted diblock copolymer films
}

Article

Accepted Version

O'Driscoll, B. M. D., Griffiths, G. H., Matsen, M. W. and Hamley, I. W. (2011) Structure variation and evolution in microphase-separated grafted diblock copolymer films. Macromolecules, 44 (21). pp. 8527-8536. ISSN 0024-9297 doi: https://doi.org/10.1021/ma2010074 Available at https://centaur.reading.ac.uk/24466/

It is advisable to refer to the publisher's version if you intend to cite from the work. See Guidance on citing.

To link to this article DOI: http://dx.doi.org/10.1021/ma2010074

Publisher: American Chemical Society

All outputs in CentAUR are protected by Intellectual Property Rights law, including copyright law. Copyright and IPR is retained by the creators or other copyright holders. Terms and conditions for use of this material are defined in the End User Agreement.

www.reading.ac.uk/centaur 
Central Archive at the University of Reading

Reading's research outputs online 


\title{
Template for Submission of Manuscripts to American Chemical Society Journals
}

\author{
Word 2003, Version 2 (2009)
}

This template is to be used to prepare manuscripts for submission to any American Chemical Society (ACS) primary research journal. As a result, it contains paragraph styles that may not normally be used in the journal you have selected for submission. Please consult the Instructions to Authors or a recent issue of the ACS journal where you plan to submit this paper for the appropriate paragraph styles. Use of this template is a benefit to the author in that the entire manuscript (text, tables, and graphics) may be submitted in one file. Inserting graphics and tables close to the point at which they are discussed in the text of the manuscript can also be a benefit for the reviewer. Use of the template is not a requirement for submission.

When you submit a manuscript using this template, you will not actually see the page formatting that appears in the printed journal. This will occur as part of the editorial production process using the paragraph tags you inserted from the template. Please read the general instructions given below on how to use the template. If you are unfamiliar with the use of templates, additional instructions can be found at the site where you downloaded this template.

\section{Using the template}

1. Abbreviated instructions for using the template follow. Additional instructions can be found in the readme file at the site where you downloaded this template.

2. If typing your manuscript directly into the template, select (highlight) the text of the template that you want to replace and begin typing your manuscript (i.e., select the Title section for typing in your title).

3. If you have already prepared your document in a Word file, you will need to attach the template to your working document in order to apply the Word Style tags. Further instructions can be found in the readme file at the site where you downloaded this template.

a. Go to the Word Style list on the formatting toolbar and you will see all the Word Styles from the template that have now been imported into the current document. A Styles toolbar has been generated that will display the different Styles for you to choose from. If this is not present, select View, Toolbars, and then select Styles and it should appear. You can close this at any time and then reopen it when needed.

b. Click in the sentence or paragraph and then go to the Word Style menu on the toolbar and select the relevant Word Style. This will apply the Word Style to the entire text (sentence or paragraph). Do this for all sections of the manuscript.

4. In ACS publications there are many different components of a manuscript (i.e., title, abstract, main text, figure captions, etc.) that are represented in the template. See the Guide, Notes, Notice, or Instructions for Authors that appear in each publication's first issue of the year and the journal's homepage to determine which parts should be included for the manuscript that you are preparing.

5. To insert graphics within the text or as a figure, chart, scheme, or table, create a new line and insert the graphic where desired. If your graphic is not visible, ensure that the Word Style is "Normal" with an automatic height adjustment. If the size of the artwork needs to be adjusted, re-size the artwork in your graphics program and repaste the artwork into the template (maximum width for single-column artwork, $3.3 \mathrm{in} .(8.5 \mathrm{~cm})$; maximum width for double-column artwork, $7 \mathrm{in} .(17.8 \mathrm{~cm})$ ). NOTE: If you are submitting your paper to a journal that requires a Table of Contents graphic, please insert the graphic at the end of the file.

6. Delete all sections from the template that are not needed, including these instructions.

7. Save the file with the graphics in place: select Save As (File menu) and save it as a document file (.doc).

8. Proof a printout of the manuscript (from a $600 \mathrm{dpi}$ or higher laser printer) to ensure that all parts of the manuscript are present and clearly legible.

9. Consult the Info for Authors page from the home page of the ACS journal that you have selected for the latest instructions on how to proceed with the submission of your manuscript.

10. Ensure that page numbers are present on all pages before submitting your manuscript. 


\section{Structure Variation and Evolution in Microphase}

\section{Separated Grafted Diblock Copolymer Films}

Benjamin M.D. O'Driscoll ${ }^{*}$, Guy H. Griffiths ${ }^{2}$, Mark W. Matsen ${ }^{2}$, and Ian W. Hamley ${ }^{1}$

${ }^{1}$ Department of Chemistry, University of Reading, Whiteknights, Reading, RG6 6AD, UK

${ }^{2}$ Department of Mathematics, University of Reading, Whiteknights, Reading, RG6 6AX, UK

Email: b.odriscoll@reading.ac.uk

RECEIVED DATE (to be automatically inserted after your manuscript is accepted if required according to the journal that you are submitting your paper to) 
ABSTRACT The phase behavior of grafted d-polystyrene-block-polymethyl methacrylate diblock copolymer films is examined, with particular focus on the effect of solvent and annealing time. It was observed that the films undergo a two-step transformation from an initially disordered state, through an ordered meta-stable state, to the final equilibrium configuration. It was also found that altering the solvent used to wash the films, or complete removal of the solvent prior to thermal annealing using supercritical $\mathrm{CO}_{2}$, could influence the structure of the films in the meta-stable state; though the final equilibrium state was unaffected. To aid in the understanding to these experimental results, a series of self-consistent field theory calculations were done on a model diblock copolymer brush containing solvent. Of the different models examined, those which contained a solvent selective for the grafted polymer block most accurately matched the observed experimental behavior. We hypothesize that the structure of the films in the meta-stable state results from solvent enrichment of the film near the film/substrate interface in the case of films washed with solvent, or faster relaxation of the non-grafted block for supercritical $\mathrm{CO}_{2}$ treated (solvent free) films. The persistence of the meta-stable structures was attributed to the slow reorganization of the polymer chains in the absence of solvent. 


\section{Introduction}

Grafted polymer films (otherwise known as polymer brushes) have garnered much attention due to their desirable physical properties relative to conventional non-grafted films (including improved mechanical robustness and prevention of dewetting), giving grafted films great potential as surface coatings. ${ }^{1-3}$ Alongside this, advances in polymerization techniques, such as polymerization from surface bound initiators, ${ }^{4,5}$ and functionalisation chemistry, including various 'click' reactions, ${ }^{6,7}$ have allowed for great diversity in the types of films that can be produced.

Grafted diblock copolymer films are a particularly interesting sub-set of these films. It is well known that block copolymers can undergo microphase separation to form a number of structures with periodicities on the order of tens of nanometers, and this makes them candidates for the next generation of sensors, membranes or lithographic templates. ${ }^{2,8}$

Recently, the phase diagram of dry (i.e. solvent free) diblock copolymer brushes (DCBs) has been simulated using self-consistent field theory (SCFT). ${ }^{9}$ This model determined that four phases may be formed: hexagonal, stripe, inverse hexagonal and uniform (Figure 1).
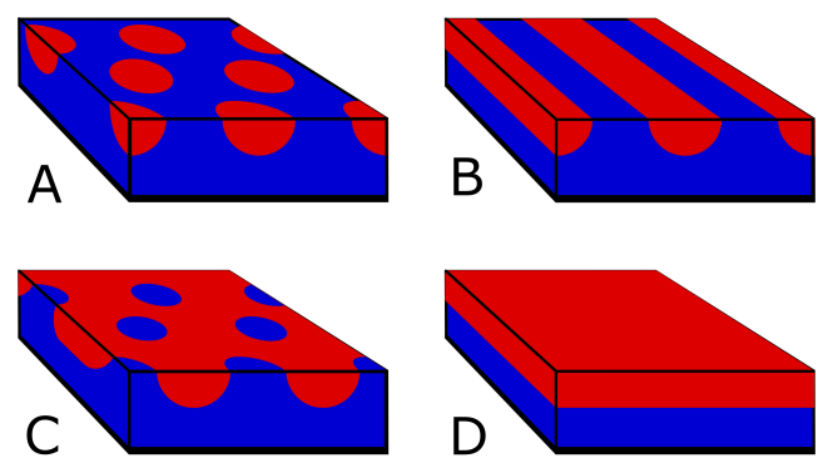

Figure 1. The four phases formed by microphase separated DCBs (A) hexagonal, (B) stripe, (C) inverse hexagonal, and (D) uniform. The non-grafted block is coloured red and the grafted block blue.

This is fewer than observed with bulk block copolymers systems due to the fact that microphase separation in these films is effectively two-dimensional. The confinement of the polymer also introduces two additional variables into the phase space. As with bulk block copolymers, the phase 
behavior of DCBs is dependent on the molecular weight of the polymer $\left(M_{n}\right)$, the composition $(f)$ and the Flory-Huggins interaction parameter $(\chi),{ }^{8}$ however, DCBs also show a dependence on both the grafting density $(\sigma)$ and the relative surface tensions of the two blocks $(\Delta \gamma) .^{9}$

These variables are often expressed in molecular weight independent terms to allow for comparison between different polymer films, with $\chi N, \Lambda N$ and $L / a N^{1 / 2}$ typically being used to describe the interaction strength, surface tension and grafting density contributions respectively. ${ }^{10}$ Here $N$ is the degree of polymerization, $\Lambda$ is a term proportional to $\Delta \gamma,{ }^{9} L$ is the measured film thickness and $a$ is the mean free end-to-end length. The film is also usually discussed in terms of an upper (non-grafted) polymer block, $A$, and a lower (grafted) block, $B$. Unlike the bulk phase diagram, the dry brush phase diagram is noticeably asymmetric; it also displays strong dependences on both $\sigma$ and $\Delta \gamma^{9,10}$

In a recent publication we described the first attempt to compare the experimental morphology with the dry brush theoretical model. ${ }^{10}$ In that study we observed all four phases, demonstrating a qualitative agreement with the model; however, we also found that there were substantial quantitative differences between the experimental and theoretical phase behavior. ${ }^{10}$ It was subsequently concluded that the most probable cause of this variation was the retention of solvent in the films.

In the bulk state, the effect of solvent has been examined from both a theoretical and experimental perspective. ${ }^{11-14}$ At low solvent volumes the polymer is plasticized by the solvent leading to an increase in the rate of polymer chain diffusion while concomitantly decreasing the glass transition temperature $\left(T_{g}\right)$. For block copolymers additional consideration needs to be given to the strength of interaction between the solvent and the two polymer blocks. With a neutral solvent (i.e. a good solvent for both blocks) the phase diagram is largely unchanged, however, the solvent improves the miscibility of the two polymer blocks leading to an effective decrease of $\chi$ with increasing solvent content. ${ }^{15,16}$ In the dilution approximation, $\chi$ decreases linearly with the volume fraction of polymer, ${ }^{15}$ and such a correlation has been experimentally shown to accurately predict shifts in the order-order transitions (OOT), though it is less accurate with predicting shifts in the order-disorder transition (ODT). ${ }^{16,17}$ If the 
solvent used shows a preference for one block over the other then the first block will be swollen more than the second. This will cause an increase in the relative volume fraction of the preferred block and correspondingly shift the expected phase boundaries. ${ }^{11}$

In this report the effect of solvent on microphase separation in DCBs is examined using a range of solvents. The films were prepared using d-polystyrene-block-poly(methyl methacrylate), $\mathrm{dPS}_{\mathrm{x}} \mathrm{PMMA}_{\mathrm{y}} \mathrm{OH}$, copolymers, where $\mathrm{x}$ and $\mathrm{y}$ define the approximate size of each block (in $\mathrm{kDa}$ ) and the $\mathrm{OH}$ represents the terminal hydroxyl group through which the polymers are grafted to the surface.. The films were subsequently treated using a number of pure and mixed solvents. Specifically, toluene, tetrahydrofuran (THF), dichloromethane (DCM) and ethyl acetate were the pure solvents used, all of which are good solvents for both polymer blocks. The mixed solvent used was a 1:1 v/v mixture of cyclohexane and acetone; individually each of these is non-solvent for one of the blocks, ${ }^{10,18,19}$ however, the mixture itself is a good solvent for the copolymers used. Solvent free films were also prepared through treatment with supercritical $\mathrm{CO}_{2}\left(\mathrm{scCO}_{2}\right) ; \mathrm{scCO}_{2}$ is a poor solvent for dPS and PMMA, but it is known to swell both polymers and subsequently will remove excess solvent from the film. ${ }^{20,21}$ In addition, the effect of the annealing process (either thermal or solvent annealing) and annealing time on structure of the films was examined

Finally, we have extended our initial SCFT calculations that supported the solvent effect hypothesis. The results of these models are compared to the observed experimental results. 


\section{Experimental}

The hydroxyl-terminated diblock copolymers were purchased from PSS (Polymer Standards Service; Mainz, Germany) and used without further purification. Polished silicon wafers were purchased from Prolog Semicor Ltd (Ukraine). These were cleaned with a Piranha solution $\left(70 \% \mathrm{H}_{2} \mathrm{SO}_{4}, 30 \% \mathrm{H}_{2} \mathrm{O}_{2}\right)$ at $90{ }^{\circ} \mathrm{C}$ for 1 hour, washed with water and ethanol then dried using a stream of $\mathrm{N}_{2}$ gas. (Caution: Piranha solution can react violently with organic compounds.) AR grade solvents were obtained from Fisher.

Relatively thick $(\sim 70 \mathrm{~nm})$ films of the polymer were prepared by spin-coating $2 \mathrm{wt} \%$ solutions (in toluene) of the polymer at $2000 \mathrm{rpm}$ onto cleaned silicon wafers. The films were annealed at $180{ }^{\circ} \mathrm{C}$, under vacuum, for 24 hours to graft the polymer chains to the surface. After cooling the wafer was cut into fragments approximately $1 \mathrm{~cm}^{2}$ in size. The excess (non-grafted) polymer was removed from each fragment by sonication in the desired solvent $(2 \times 10$ min with $5 \mathrm{~mL}$ of solvent) followed by rinsing of each fragment with $\sim 1 \mathrm{~mL}$ of the same solvent. The films were dried using a stream of $\mathrm{N}_{2}$ gas.

One set of toluene washed films were subsequently washed with $\mathrm{scCO}_{2}\left(35{ }^{\circ} \mathrm{C}\right.$ and 3000 psi for 30 $\min )$ to remove the excess solvent.

Microphase separation was induced in the films by either annealing them for a second time at $180{ }^{\circ} \mathrm{C}$ under vacuum for a period of 6 to 192 hours, or by exposure to a saturated atmosphere of solvent for 24 hours.

AFM images were collected with a Veeco Explorer with a $2 \mu \mathrm{m}$ scanner in non-contact mode. AFM tips (Veeco) with $k=20-80 \mathrm{~N} / \mathrm{m}$ and $f_{0}=130-320 \mathrm{kHz}$ were used with a set-point ratio of $50-60 \%$.

Ellipsometry measurements were performed at the University of Surrey, UK, on a J.A Woollam ellipsometer. The optical constants were measured using an uncoated silicon wafer, while the polymer films were modeled as a single Cauchy layer. However, due to the small thicknesses of the grafted films the values of the Cauchy coefficients were fixed at $a=1.52$ and $b=0.001$. When errors are reported these represent the standard deviation across five measurements. 
Gas chromatography-mass spectroscopy measurements (GCMS) were performed using a CPPoraBond Q fused silica column (25 m x $0.25 \mathrm{~mm}$; Varian) in a Trace GC Ultra GCMS (Thermo Scientific). The carrier gas (helium) flow rate was $1.2 \mathrm{~mL} / \mathrm{min}$ and the temperature was ramped from 40 ${ }^{\circ} \mathrm{C}$ to $280{ }^{\circ} \mathrm{C}$ at $15^{\circ} \mathrm{C} / \mathrm{min}$. The samples were prepared by immersing a grafted film (previously toluene washed and dried) into a known amount of DCM for $15 \mathrm{~min}$ and comparing the measured total ion current from the toluene peak with previously prepared standards. THF was used as the internal reference for the system. Two sets of measurements were done, one using DCM with a known amount of toluene already present and one without. These gave similar results and all six runs were averaged to give the reported results.

Our SCFT calculations utilize the unit-cell approximation described in a recent paper by Griffiths et al., ${ }^{22}$ generalized to include solvent. ${ }^{15}$ 


\section{Results}

For this work two dPS $\mathrm{PMMA}_{y} \mathrm{OH}$ polymers were used to prepare DCBs (table 1). These were attached to the substrate through terminal groups on the PMMA block leaving the smaller dPS block as the non-grafted block. A single large sample of each polymer was prepared initially before being divided into fragments prior to the removal of the excess, non-grafted, polymer; this was done to ensure the greatest homogeneity across the samples.

Ellipsometry measurements were performed on the dry films to determine the film thickness (following 6 hours of thermal annealing), and from these results $\sigma$ and $L / a N^{1 / 2}$ values were calculated using known values for the monomer volume and $a$ (table 1$){ }^{23}$ The measured values for $L$ reported here are similar to those previously reported for these polymers, ${ }^{10}$ and were found to be independent of the washing solvent used within the error of the measurement. Moreover, the calculated values of $L / a N^{1 / 2}$ are suitably close to the value of 0.5 used both here and in previous SCFT models. ${ }^{9}$

Table 1. Parameters of the polymer films.

\begin{tabular}{lccccccccc}
\hline Polymer & $M_{n}$ & $P D I^{\dagger}$ & $f_{P S}$ & $\chi N$ & $\Lambda N$ & $R_{0}$ & $L$ & $L / a N^{1 / 2}$ & $\sigma$ \\
& $\mathrm{kg} / \mathrm{mol}$ & & & & & $\mathrm{nm}$ & $\mathrm{nm}$ & & $\mathrm{nm}^{-2}$ \\
\hline $\mathrm{dPS}_{47} \mathrm{PMMA}_{135} \mathrm{OH}$ & 182.0 & 1.14 & 0.24 & 65.4 & 1.60 & 27.6 & 11.5 & 0.42 & 0.043 \\
$\mathrm{dPS}_{62} \mathrm{PMMA}_{69} \mathrm{OH}$ & 131.0 & 1.07 & 0.45 & 46.0 & 1.38 & 23.3 & 12.5 & 0.54 & 0.065 \\
\hline
\end{tabular}

${ }^{\dagger} P D I$ denotes the polydispersity index.

To determine the volume of solvent present in the dried but unannealed films both ellipsometry and GCMS measurements were performed on toluene washed films. The resulting calculated volume fractions of solvent in the measured films are reported in table 2.

Table 2. Solvent volume in toluene washed films 


\begin{tabular}{lcc}
\hline & \multicolumn{2}{c}{$\%$ Toluene } \\
\hline Polymer & Ellipsometry & GCMS \\
\hline $\mathrm{dPS}_{47} \mathrm{PMMA}_{135} \mathrm{OH}$ & $19 \pm 7$ & $6 \pm 2$ \\
$\mathrm{dPS}_{62} \mathrm{PMMA}_{69} \mathrm{OH}$ & $9 \pm 3$ & $3 \pm 1$ \\
\hline
\end{tabular}

The GCMS results qualitatively demonstrate that some solvent remains in the films even after drying with a stream of $\mathrm{N}_{2}$ gas, though there is disagreement between the two techniques in qualitative terms and all of the measured values have large errors associated with them. It is likely that some of the variation between the two techniques can be attributed to the underlying assumptions made when analyzing the results. For the ellipsometry it was assumed that the refractive index of the polymer film was constant and that there was no difference in the optical constants associated with the substrate (see experimental section). This left the film thickness as the only variable to be fitted, with the measured change in film thickness assumed to be proportional to the volume fraction of solvent in the washed film. For the GCMS measurements it was assumed that the washing procedure efficiently removed all of the target from the film, such that the measured amount of toluene in the carrier solvent could be directly compared to the toluene content of the films. Both sets of assumptions have the potential to introduce error into the final measurement. Unfortunately, improving the quality of these measurements is difficult due to the very low volumes of solvent incorporated in the films and it is not possible to conclude which set of numbers are most accurate. Nevertheless, the above results are consistent with similar experiments carried out on non-grafted polymer films, with both Perlich et al. ${ }^{24}$ and GarciaTuriel and Jerome ${ }^{25}$ observing that there is a significant amount of solvent trapped in the film measured to be between $8-14 \%$ for toluene in thin polystyrene films. ${ }^{24}$ Residual solvent was also observed in the films even after relatively long periods of heating, suggesting that the interaction between the polymer and solvent is strong. ${ }^{24}$ These results are supported by other studies, ${ }^{26}$ though a recent publication claimed no solvent was present. ${ }^{27}$ It has also been reported that there is an additional 
strong interaction between the solvent and the substrate in certain systems (including in polymer films on silicon wafers). ${ }^{13,24,25}$

Interestingly, both the ellipsometry and GCMS data shows a much larger toluene concentration in the $\mathrm{dPS}_{47} \mathrm{PMMA}_{135} \mathrm{OH}$ films. While the exact cause of this is unknown, or indeed how significant the difference is given the errors associated with the measurements, it is possible that this variation is due to the lower grafting density of the $\mathrm{dPS}_{47} \mathrm{PMMA}_{135} \mathrm{OH}$ films. A larger distance between grafting points should allow for greater interpenetration of solvent and this may, in turn, lead to higher solvent retention.

The assumption in the ellipsometry that the refractive index of the solvent and polymer is the same will clearly not be valid for a film expanded with $\mathrm{scCO}_{2}$. Therefore, to examine the effect that $\mathrm{scCO}_{2}$ will have on DCBs a test was done using a thick (effectively non-grafted) $\mathrm{dPS}_{62} \mathrm{PMMA}_{69} \mathrm{OH}$ film. Post $\mathrm{scCO}_{2}$ treatment the film was determined to have a thickness of $80.9 \mathrm{~nm}$, however, following thermal annealing (for 6 hours at $180^{\circ} \mathrm{C}$ ) it was observed that the film thickness had decreased to $73.2 \mathrm{~nm}$. This result indicates that, like the grafted films washed with solvent, those treated using $\mathrm{scCO}_{2}$ should undergoes a volume contraction during thermal annealing.

\section{Solvent Annealing}

Solvent annealing has previously been used to good effect to facilitate the formation of microphase separated structures in non-grafted diblock copolymer films, ${ }^{28}$ with microphase separated structures being formed after relatively short annealing times. ${ }^{29}$ However, the same experiment performed on the DCBs examined here was far less successful. As can be seen in figure 2 there is only weak microphase separation in some of the films (with ethyl acetate showing the strongest segregation) following a 24

hour solvent annealing period. (Note: PMMA appears as the brighter phase in the AFM phase images. ${ }^{30}$ The topographic images corresponding to the phase images present below are provided in the supporting information) 


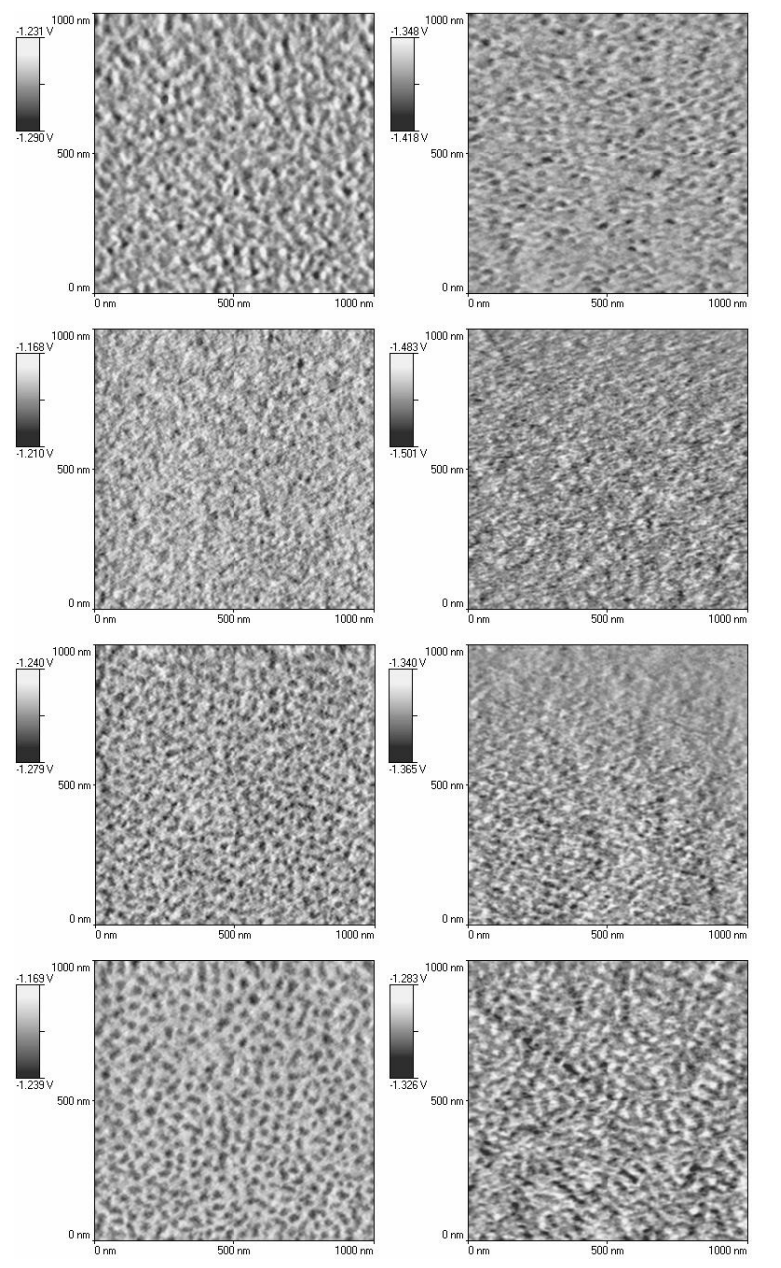

Figure 2. AFM phase images of $\mathrm{dPS}_{47} \mathrm{PMMA}_{135} \mathrm{OH}$ (left) and $\mathrm{dPS}_{62} \mathrm{PMMA}_{69} \mathrm{OH}$ (right) solvent annealed with toluene ( $1^{\text {st }}$ row $)$, THF ( $2^{\text {nd }}$ row $), \operatorname{DCM}\left(3^{\text {rd }}\right.$ row $)$, and ethyl acetate $\left(4^{\text {th }}\right.$ row $)$.

For the film displaying noticeable microphase separation the structures are relatively disordered over long length scales when compared with non-grafted films. However, similarly low ordered structures have recently been produced by both single-chain-in-mean-field ${ }^{31}$ and dissipative particle dynamics simulations $^{32}$ of DCB films in the presence of solvents.

The most probable cause for the lack of microphase separation in these samples is the reduction in the magnitude of $\chi$ (in line with the dilution approximation) caused by presence of the solvent. The theoretical model for dry DCBs shows that the critical $\chi N$ value below which only the uniform phase is formed is relatively high when compared to the equivalent ODT critical point in the bulk phase diagram, ${ }^{9}$ making DCBs more sensitive to dilution with solvent. This issue is discussed further in the section relating to the computer modeling results, below. 
From a theoretical perspective, inclusion of solvent into a DCB yields a ternary system, for which three separate interaction parameters are required: one for the polymer-polymer interaction $\left(\chi_{A B}\right.$, equivalent to $\chi$ for the dry brush) and two polymer-solvent interactions $\left(\chi_{A S}\right.$ and $\left.\chi_{B S}\right)$. It is the magnitude of these latter two that determines the uptake of solvent by the respective polymer blocks.

The magnitude of $\chi_{A S}$ and $\chi_{B S}$ can be approximated using the solubility parameter for materials involved; we have used the values given by Hansen (table 3$){ }^{33}$

Table 3. The calculated interaction parameters and physical constants for the solvents used in this work.

\begin{tabular}{lccccc}
\hline & \multicolumn{3}{c}{ Interaction Parameters $^{2}$} & Physical Parameters $^{\dagger}$ \\
\hline & $\chi_{\text {PS-Solv }}$ & $\chi_{\text {PMMA-Solv }}$ & Selectivity* & b.p. & $P_{V}$ \\
\hline Toluene & 0.062 & 0.106 & PS & 110 & 22 \\
THF & 0.066 & 0.026 & PMMA & 66 & 143 \\
DCM & 0.023 & 0.011 & PMMA & 40 & 353 \\
Ethyl Acetate & 0.028 & 0.023 & PMMA & 77 & 73 \\
Cyclohexane & 0.121 & 0.167 & PS & 81 & 77 \\
Acetone & 0.104 & 0.026 & PMMA & 56 & 184 \\
\hline
\end{tabular}

${ }^{\dagger}$ b.p. is the boiling point $\left(\right.$ in ${ }^{\circ} \mathrm{C}$ ) and $P_{V}$ is the vapor pressure (in $\mathrm{mmHg}$ at $20{ }^{\circ} \mathrm{C}$ ). ${ }^{*}$ The polymer block that each solvent most positively interacts with.

Larger values of $\chi_{A S}$ and $\chi_{B S}$ indicate that the two components are less miscible. From the above values it would therefore be expected that, of the pure solvents, toluene and THF should swell the films least and, by application of the dilution approximation, be most likely to produce microphase separated films. This is evidently not the situation observed in the above AFM images and so direct comparison of the interaction parameters for different systems is not useful. Nevertheless, as is demonstrated later, the relative selectivity of the solvents does seem to correlate with the experimental results.

Possible reasons for the lack of direct comparability between the calculated solubility parameters include: the application of bulk solubility parameter to thin films, use of generic values for the polymers 
(which were non-deuterated homopolymers), inaccuracies when $\chi$ is relatively high, ${ }^{33}$ and the reduced degrees of freedom of the polymer in grafted films.

For films washed with solvent then quickly dried, the AFM images did not show any non-uniform phase structures, regardless of the solvent used (results not shown). This drying process is equivalent to the rapid drying experienced during spin-coating, a process that frequently produces microphase separated structures. ${ }^{34}$ The absence of similar structures in these films points to additional constraints acting on the film.

In line with the dilution approximation, dissolving of the copolymer in solvent, be it free in solution or as an immersed grafted film, will prevent microphase separation. Upon rapid drying of the solvent microphase separated structures will only be observed if the rate of polymer diffusion is commensurate with or faster than the rate of solvent loss. Based on the solvent annealing results, microphase separation would only be expected in the ethyl acetate and DCM washed films, however, even with these films no structure is observed. It is apparent therefore that reorganization of the polymer chains in the grafted films is relatively slow when compared with non-grafted films, leaving the grafted films in a kinetically trapped disordered state. (Note: for grafted films, there is no theoretical difference between the uniform state (figure 1) and a non-equilibrium or disordered state due to the inherent alignment of the polymer chains away from the surface).

\section{Thermal Annealing}

\section{Short Annealing Periods}

As was previously observed, ${ }^{10}$ annealing for a short period of time at high temperatures and low pressures results in strong microphase separation within these films (figure 3). Ordering was observed in as little as three hours, which is slightly slower than the speed of microphase separation observed with non-grafted films. ${ }^{35}$ For all of the films treated with neat solvents, those prepared from $\mathrm{dPS}_{47} \mathrm{PMMA}_{135} \mathrm{OH}$ show hexagonal-type ordering, while those prepared from $\mathrm{dPS}_{62} \mathrm{PMMA}_{69} \mathrm{OH}$ show inverse hexagonal-type ordering. 

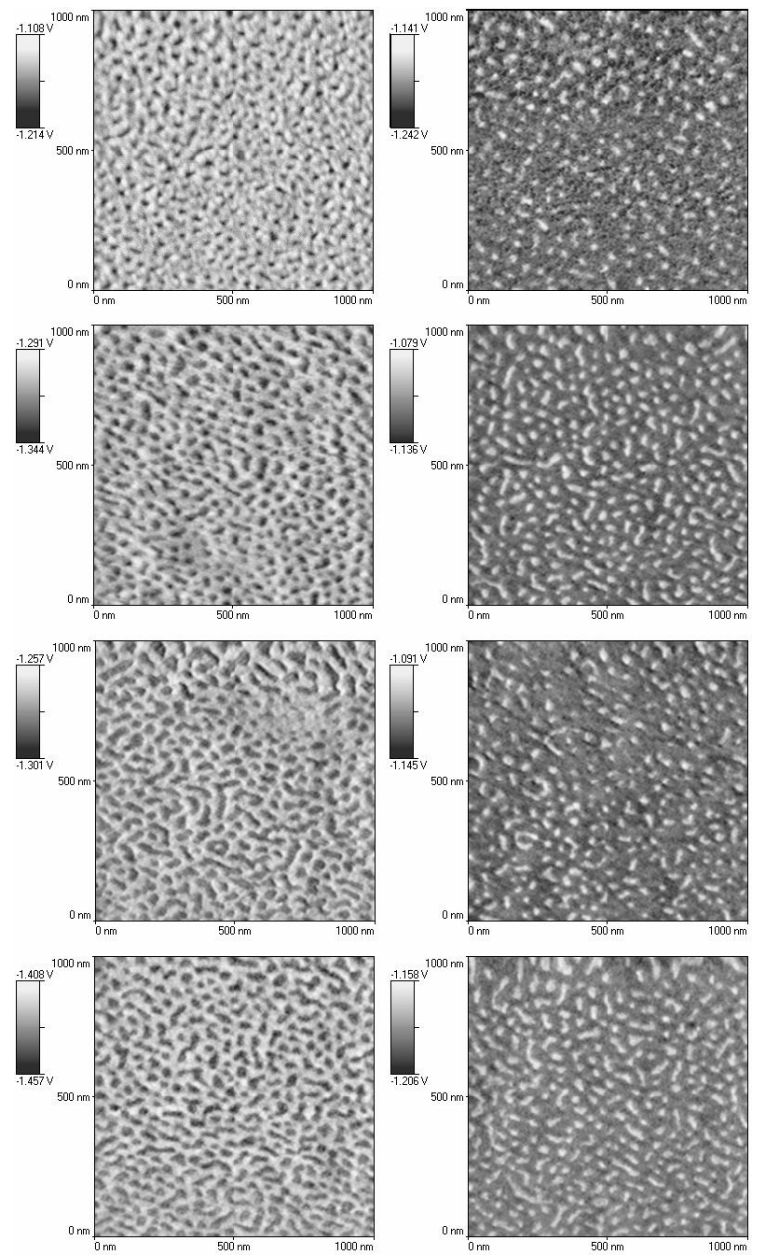

Figure 3. AFM phase images of $\mathrm{dPS}_{47} \mathrm{PMMA}_{135} \mathrm{OH}$ (left) and $\mathrm{dPS}_{62} \mathrm{PMMA}_{69} \mathrm{OH}$ (right) thermally annealed at $180^{\circ} \mathrm{C}$ for 6 hours after washing with toluene ( $1^{\text {st }}$ row $)$, THF ( $2^{\text {nd }}$ row), DCM ( ${ }^{\text {rd }}$ row), and ethyl acetate $\left(4^{\text {th }}\right.$ row $)$.

However, within these assignments there is a noticeable solvent effect on the shape of the dPS and PMMA domains. The magnitude of the shape anisotropy was determined by calculating the circularity, Circ., of the minor domains in the AFM images; where Circ. $=4 \pi[$ Area/Circumference $]$, with the area and circumference being determined using image analysis. At the same time the periodicity of the films was determined using fast Fourier transform analysis (table 4).

Table 4. Circularity and periodicity values for the $6 \mathrm{hr}$ annealed films 


\begin{tabular}{lcccc}
\hline & \multicolumn{2}{c}{$\mathrm{dPS}_{47} \mathrm{PMMA}_{135} \mathrm{OH}$} & $\mathrm{dPS}_{62} \mathrm{PMMA}_{69} \mathrm{OH}$ \\
& Circ. & Period. & Circ. & Period. \\
& & $\mathrm{nm}^{-1}$ & & $\mathrm{~nm}^{-1}$ \\
\hline Toluene & 0.79 & 48 & 0.78 & 48 \\
THF & 0.81 & 44 & 0.82 & 48 \\
DCM & 0.70 & 48 & 0.79 & 48 \\
Ethyl Acetate & 0.71 & 44 & 0.76 & 48 \\
scCO 2 & 0.73 & 48 & 0.71 & 53 \\
Cyclohexane & 0.77 & 50 & 0.64 & 46 \\
/Acetone & & & & \\
\hline
\end{tabular}

Of the neat solvents, the ethyl acetate treated films had the greatest anisotropy for both $\mathrm{dPS}_{47} \mathrm{PMMA}_{135} \mathrm{OH}$ and $\mathrm{dPS}_{62} \mathrm{PMMA}_{69} \mathrm{OH}$, while THF treated films had the least anisotropy. Notably, although the solvent content of the annealed films was not examined here, it is reasonable to assume, based on the work of Perlich et al., ${ }^{24}$ that annealing the films at $180{ }^{\circ} \mathrm{C}$ for 6 hours would be sufficient to remove the vast majority of the solvent.

The anisotropy trends observed with these films is somewhat unusual in that for the $\mathrm{dPS}_{47} \mathrm{PMMA}_{135} \mathrm{OH}$ films it is the circularity of the dPS domains being measured, while in the $\mathrm{dPS}_{62} \mathrm{PMMA}_{69} \mathrm{OH}$ films it is the PMMA domains. For the systems treated with ethyl acetate (and to a lesser extent with DCM) the observed elongation of the domains indicates that these films are almost in the stripe phase. That both of the ethyl acetate treated films are showing this indicates that the stripe phase has an expanded footprint in the phase space due to the presence of the solvent.

It should also be noted that the phases formed here are slightly different to the predicted dry brush phases (figure 1), as the corresponding topographic images for the above phase images indicate that the dPS domains are higher than the PMMA domains (supporting information available (figures III and IV)).

Correlations between the height and phase shift in tapping mode AFM have been observed before and, in part, the observed height variation can be attributed to stiffness variation across the surface. ${ }^{36}$ 
However, Wang et al. (who examined non-grafted block copolymer films) have shown that the topographic variation is generally real. ${ }^{37}$

\section{Long Annealing Periods}

The microphase separated structures observed after a 6 hour annealing period showed good long term stability at room temperature, with the structures being unchanged more than four months after the 6 hour annealing process. However, further annealing at high temperatures and low pressures led to a gradual but dramatic loss of structure in all the films examined here (figure 4). It is clear, therefore, that the microphase separated structures shown in figure 3 are not equilibrium dry DCB structures. The formation of meta-stable structures upon drying of the film has previously been noted in non-grafted films, ${ }^{35}$ but the structure change observed here in moving from this meta-stable state to the final equilibrium state are much more pronounced.

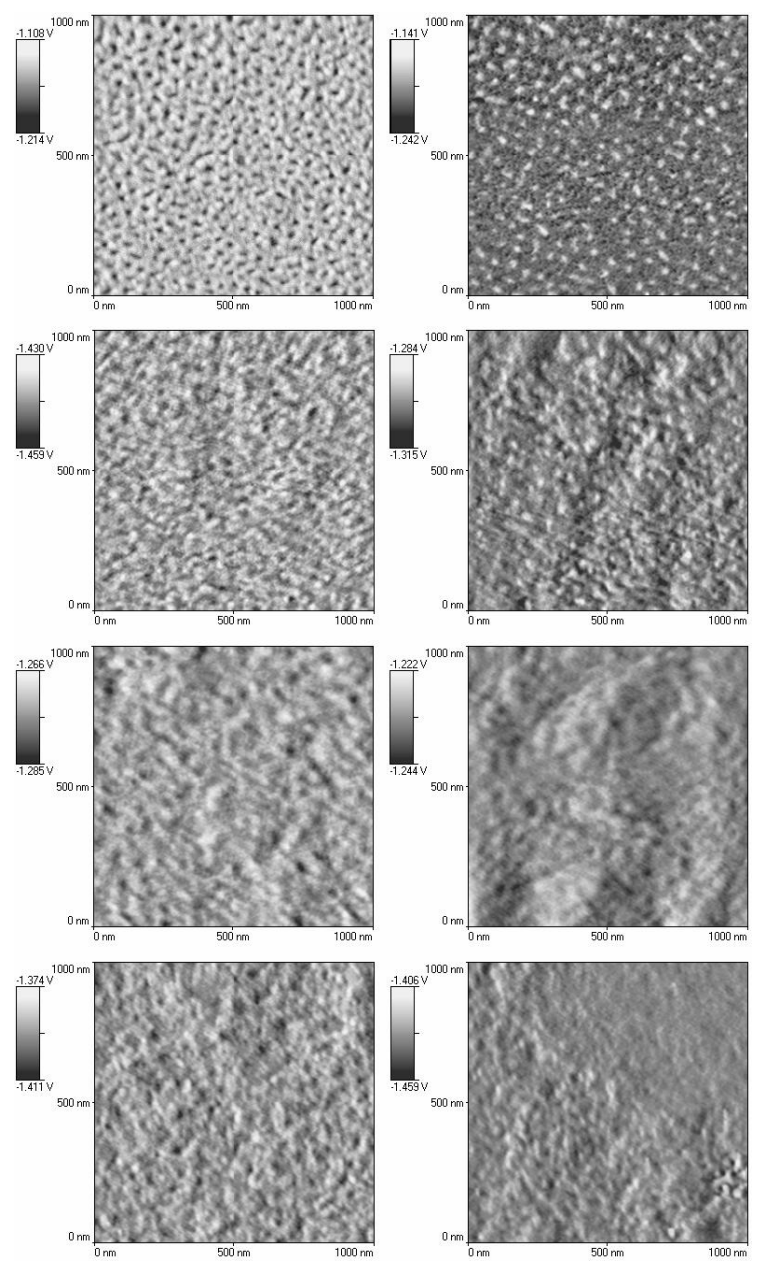


Figure 4. AFM phase images of $\mathrm{dPS}_{47} \mathrm{PMMA}_{135} \mathrm{OH}$ (left) and $\mathrm{dPS}_{62} \mathrm{PMMA}_{69} \mathrm{OH}$ (right) washed with toluene and then thermally annealed at $180{ }^{\circ} \mathrm{C}$ for 6 hours $\left(1^{\text {st }}\right.$ row $), 24$ hours $\left(2^{\text {nd }}\right.$ row $), 96$ hours $\left(3^{\text {rd }}\right.$ row), and 192 hours $\left(4^{\text {th }}\right.$ row $)$.

Given the lack of observed microphase separation visible in the AFM images after 192 hours of annealing, it can be concluded that both of the polymers are tending towards a uniform type structure. Some of the $\mathrm{dPS}_{47} \mathrm{PMMA}_{135} \mathrm{OH}$ films exhibit a slightly mottled appearance, but the low contrast of these structures points to a near homogeneous film being present. The characteristic size of these features is also much greater than the radius of gyration of the polymer and so they cannot correspond to microphase separation. Degradation of the polymer was also eliminated as a cause of the loss of structure (results not shown).

Based on the theoretical model for the dry brush (at $\Lambda N=0$ ), it would be expected that the $\mathrm{dPS}_{62} \mathrm{PMMA}_{69} \mathrm{OH}$ films would form the uniform phase, while $\mathrm{dPS}_{47} \mathrm{PMMA}_{135} \mathrm{OH}$ would form the stripe phase. That $\mathrm{dPS}_{47} \mathrm{PMMA}_{135} \mathrm{OH}$ instead forms the uniform phase can only be explained by the surface tension difference between the two polymers. Previously published SCFT models observed that a $\Lambda N$ value of -0.15 produced a significant change in the phase diagram. ${ }^{9}$ As this $\Lambda N$ value is equivalent to a $\Delta \gamma$ of approximately $0.12 \mathrm{mN} / \mathrm{m}$, it was subsequently hypothesized that for most real systems the magnitude of $\Lambda N$ would dominate the phase behavior. ${ }^{10}$ This would indeed appear to be the case here, as the surface tension difference between the two polymers used here, at $1.0 \mathrm{mN} / \mathrm{m}\left(\gamma_{\mathrm{PS}}=40.2 \mathrm{mN} / \mathrm{m}\right.$ and $\left.\gamma_{\mathrm{PMMA}}=41.2 \mathrm{mN} / \mathrm{m}\right),{ }^{38}$ is as small as can be reasonably produced.

Relative to the 6 hour annealed films, the films annealed for 192 hour present smooth surfaces, indicating a minimization of the surface energy and supporting the conclusion that these films are at or near equilibrium.

\section{Treatment with Supercritical $\mathrm{CO}_{2}$}


Returning to the meta-stable stable ordered structures, an attempt was made to produce a solvent-free film by soaking toluene washed films in $\mathrm{scCO}_{2}$. The success of this solvent removal process could not be confirmed directly in the grafted films due to the restrictions on the ellipsometry modeling, however, the ellipsometry data on thick non-grafted films (see above) demonstrates that $\mathrm{scCO}_{2}$ swells the polymers while at the same time is capable of dissolving small molecules. ${ }^{21}$ We assume therefore that the majority of the residual solvent was removed from the films after treatment with $\mathrm{scCO}_{2},{ }^{39}$ and that the film thickness increases following the treatment process, with void spaces in the film providing the additional volume. ${ }^{40}$ The $\mathrm{scCO}_{2}$ treatment process itself did not induce microphase separation and the treated, but unannealed films presented a disordered/uniform structure (results not shown).

Upon thermal annealing, the $\mathrm{scCO}_{2}$ treated $\mathrm{dPS}_{47} \mathrm{PMMA}_{135} \mathrm{OH}$ and $\mathrm{dPS}_{62} \mathrm{PMMA}_{69} \mathrm{OH}$ films formed the hexagonal and inverse hexagonal structures respectively (figure 5), as was observed with the films washed with neat solvents. However, it is clear that the $\mathrm{scCO}_{2}$ treatment has a pronounced effect on the polymer diffusion kinetics. Although strong microphase separation is observed in the $\mathrm{dPS}_{62} \mathrm{PMMA}_{69} \mathrm{OH}$ film after a 6 hour annealing period, a much longer period of time (24 hours) was needed for the $\mathrm{dPS}_{47} \mathrm{PMMA}_{135} \mathrm{OH}$ film (supporting information available (figures VII and VIII)). The periodicities of the films are also slightly larger than those observed in the solvent washed films (table 4). Nevertheless, as with the solvent treated films, long annealing times again directed the films to form the uniform phase (supporting information available).
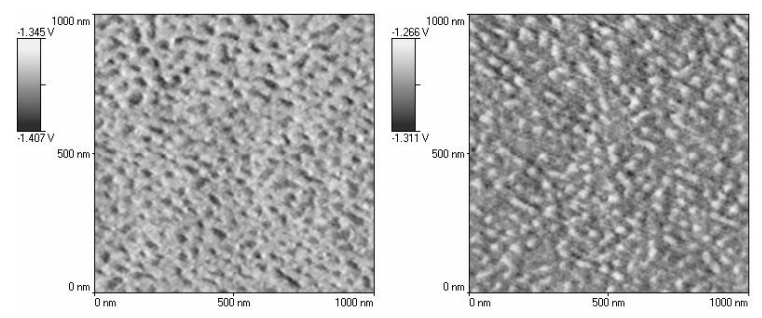

Figure 5. AFM phase images of films treated with $\mathrm{scCO}_{2}$ and annealed: (left) $\mathrm{dPS}_{47} \mathrm{PMMA}_{135} \mathrm{OH}$, annealed for 24 hours, and (right) $\mathrm{dPS}_{62} \mathrm{PMMA}_{69} \mathrm{OH}$, annealed for 6 hours. 
The formation of meta-stable microphase separated structures in the $\mathrm{scCO}_{2}$ treated films shows that these structures are not inherently dependent on the presence of a liquid solvent, though the nature of the solvent does have an impact on the structure of the film. The slow formation of the meta-stable structure in the $\mathrm{scCO}_{2}$ treated films also shows that for at least part of the annealing process enough solvent is retained in the solvent washed films to allow for faster chain diffusion. Indeed, that a metastable state forms within as little as three hours with the solvent washed films suggests that much of the reorganization occurs early in the annealing process.

\section{Mixed Solvent}

In a final experiment a number of films were washed with a 1:1 v/v mixture of cyclohexane and acetone. Like most of the neat liquid systems no significant microphase separation was observed after solvent annealing (results not shown), however, significant differences were observed with the thermally annealed films (figure 6).

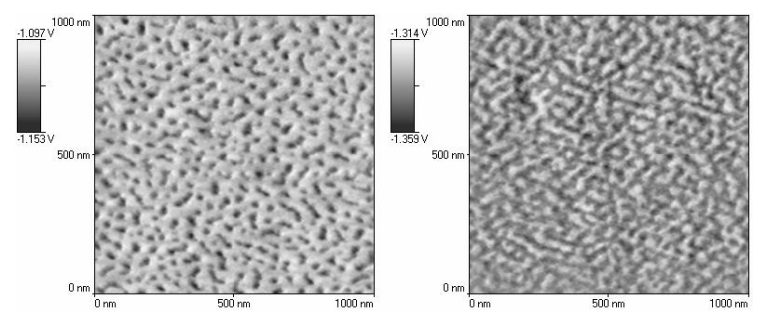

Figure 6. AFM phase images of cyclohexane/acetone washed films annealed for 6 hours (left) $\mathrm{dPS}_{47} \mathrm{PMMA}_{135} \mathrm{OH}$ and (right) $\mathrm{dPS}_{62} \mathrm{PMMA}_{69} \mathrm{OH}$.

For the $\mathrm{dPS}_{62} \mathrm{PMMA}_{69} \mathrm{OH}$ sample, the film formed the stripe phase, with the majority of the PMMA domains having lengths much greater than their widths. Subsequently, this film has the lowest average circularity of all of the samples examined, while at the same time having a decreased periodicity (table 4). For the $\mathrm{dPS}_{47} \mathrm{PMMA}_{135} \mathrm{OH}$ film the effect of the mixed solvent is not so dramatic, nevertheless, the periodicity of this sample is largest of all the $\mathrm{dPS}_{47} \mathrm{PMMA}_{135} \mathrm{OH}$ films examined here.

Given that cyclohexane is a non-solvent for PMMA and acetone a non-solvent for dPS, it is likely that the two solvents, when adsorbed into the film, will be partitioned between the two blocks; with the 
extent of partitioning will be limited by the entropic penalty associated with separating the two miscible solvents. The absence of microphase separation in the solvent annealed films suggests that the mixed solvent decreases $\chi$, in accordance with the dilution approximation, in the same manner as the neat solvents and thus the use of two solvents does not decrease the miscibility of the two blocks.

The formation of the stripe phase in one film and the moderate anisotropy of the domains in the other (table 4) indicates that there has also been an expansion of the footprint of the stripe phase in this system. The mixed solvent washed films therefore share some similarities with the ethyl acetate and DCM washed films, though here the interactions will be more complex due to the presence of two solvents.

\section{SCFT Modeling}

A range of SCFT calculations were performed on simulated DCBs at $L=0.5 a N^{1 / 2}$ and $\chi_{A B} N=50$, with the magnitudes of $\chi_{A S} N$ and $\chi_{B S} N$ being varied according to the values listed in table 5. Changing $\chi_{A S} N$ and $\chi_{B S} N$ varies the 'quality' of the solvent; with larger values corresponding to a poorer solvent for that particular polymer block. A selection of the calculated phase diagrams are also shown in figure 7 and the determined phase boundaries listed in table 5. These phase diagrams show the free energies of the different phases as a function of $f_{\mathrm{A}}$ (the fraction of the non-grafted block), with the thermodynamically favored phase being the one with the lowest free energy at a given composition.

Table 5. Modeling parameters and results

\begin{tabular}{l|c|ccc|cccc}
\hline Model & $\begin{array}{c}\text { Selective } \\
\text { for block }\end{array}$ & \multicolumn{2}{|c|}{ Interaction Parameters } & \multicolumn{3}{c}{ Phase Boundaries $\left(f_{\mathrm{A}}\right)^{*}$} \\
& & $\chi_{A B} N$ & $\chi_{A S} N$ & $\chi_{B S} N$ & $\mathrm{U} / \mathrm{H}$ & $\mathrm{H} / \mathrm{S}$ & $\mathrm{S} / \mathrm{IH}$ & $\mathrm{IH} / \mathrm{U}$ \\
\hline 1 & $\mathrm{~B}$ & 50 & 100 & 70 & 0.193 & 0.269 & 0.430 & 0.563 \\
2 & $\mathrm{~B}$ & 50 & 150 & 100 & 0.123 & 0.309 & 0.848 & 0.875 \\
3 & $\mathrm{~B}$ & 50 & 220 & 150 & 0.112 & 0.340 & $0.859^{\dagger}$ & - \\
\hline
\end{tabular}




\begin{tabular}{l|c|ccc|cccc}
\hline 4 & Neutral & 50 & 100 & 100 & - & $0.197^{\ddagger}$ & 0.244 & 0.389 \\
\hline 5 & A & 50 & 70 & 100 & - & - & - & - \\
6 & A & 50 & 100 & 150 & - & - & - & - \\
7 & A & 50 & 150 & 220 & - & - & - & - \\
\hline
\end{tabular}

* The phase abbreviations for the different phases are: uniform (U), hexagonal (H), stripe (S) and inverse hexagonal (IH). ${ }^{\dagger}$ Stripe/uniform phase boundary. ${ }^{\ddagger}$ Uniform/stripe phase boundary.
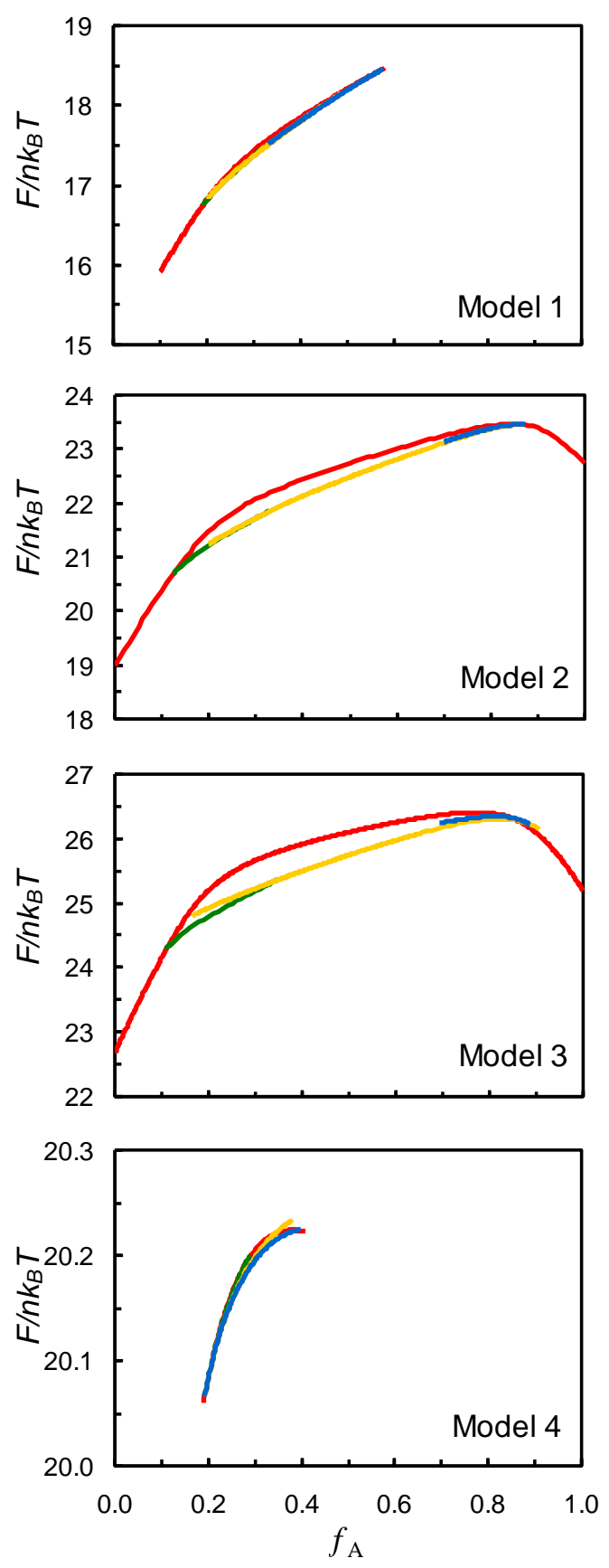
Figure 7. Free-energy plots for the Models 1, 2, 3 and 4 systems; $F$ is the free energy, $n$ is the number of polymer chains in the simulation, $k_{B}$ is Boltzmann's constant, and $T$ is the temperature The phases are coloured: uniform (red), hexagonal (green), stripe (yellow), and inverse hexagonal (blue).

These models are the theoretical analogue of a diblock copolymer film immersed in solvent. Such a system was not directly examined in these experiments, however, as is demonstrated below, the models have proved useful in explaining the behavior observed in the various films examined here. The key to this comparability is the use of different $\chi_{A S}$ and $\chi_{B S}$ values in the simulations. Increasing the magnitude of these parameters leads to a reduction in the amount of solvent incorporated into the film (figure 8), which mimics both a film that is in equilibrium with a solvent vapor (as opposed to the liquid solvent) and a film that has lost an amount of solvent through evaporation. At the extreme of very high $\chi_{A S}$ and $\chi_{B S}$ values, no solvent will be incorporated into the film, which is effectively equivalent to the case of a dry film in contact with air.
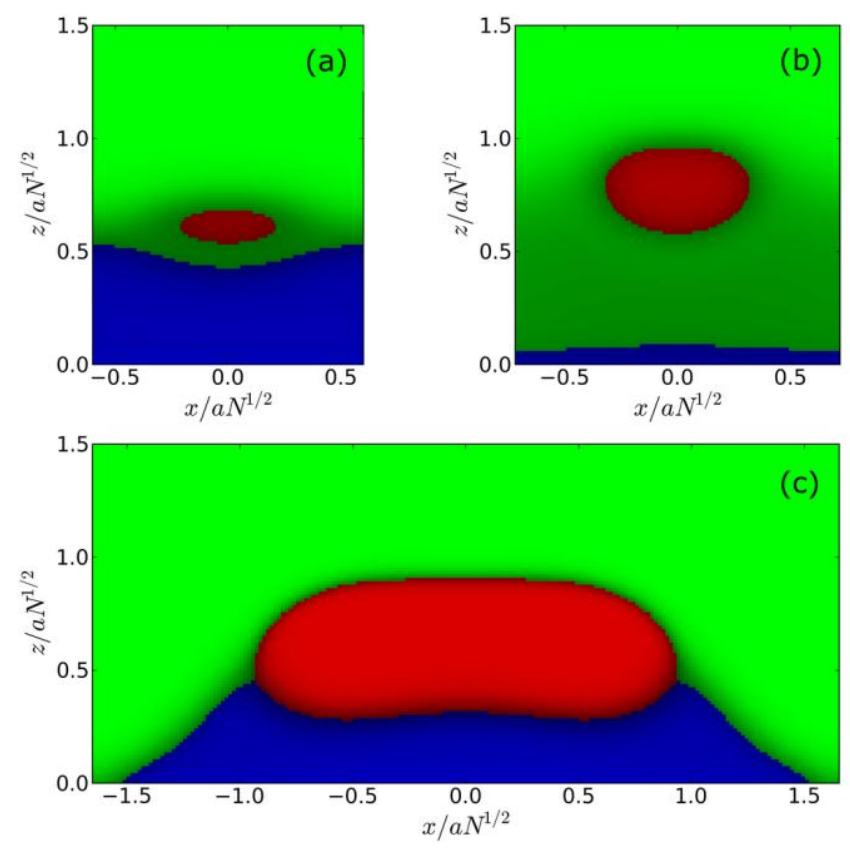

Figure 8. Transverse cross-sections of the stripe phase for (a) the Model 4 system $\left(f_{A}=0.22\right)$; (b) the Model 1 system $\left(f_{A}=0.33\right)$, and (c) the Model 2 system $\left(f_{A}=0.58\right)$. The colors correspond to: red - the non-grafted block (A), blue - the grafted block (B), and green - the solvent. Areas with deeper coloration correspond to zones of mixing between the solvent and the polymer blocks, with the 
dominant component indicated by the hue of the color. Here $x$ and $z$ are the Cartesian axes that run horizontally and vertically normal to the long-axis of a stripe phase feature.

Relative to the dry brush system, the addition of a neutral solvent does not significantly alter the footprint of the non-uniform phase region. However, as is evident in figure 8, at low $\chi_{A S} N$ and $\chi_{B S} N$ values the simulation predicts that there will be significant penetration of solvent into the film, with the highest solvent content in the interfacial region between the two block copolymers. When the solvent is made selective for the grafted block the footprint of the non-uniform phase region expands significantly. As figure 8 shows, this is accompanied by a significant alteration in the topology of the film surface. For the neutral solvent case (Model 4), there is only a slight corrugation of the surface, while for the selective solvent case (Models 1 and 2), the corrugation is predicted to increase as $\chi_{A S} N$ and $\chi_{B S} N$ increase. Such corrugation was not observed in the dry brush simulations, which produced a flat surface, ${ }^{9}$ but is consistent with the results reported here. The use of a solvent that is selective for the non-grafted block eliminates all of the non-uniform phases from the phase diagram (not shown). (Note: the expansion of the stripe phase predicted by Model 2 is slightly different to the initial phase diagram we reported previously using the same parameters, ${ }^{10}$ but this can be attributed to the greater accuracy of the models reported here.)

In addition to these changes with solvent selectivity, there is also notable variation in the free energy profiles with increasing $\chi_{A S} N$ and $\chi_{B S} N$. For all of the simulations with low $\chi_{A S} N$ and $\chi_{B S} N$ values (Models 1, 4 and 5), the free energy difference between the various phases is very small. Relative to thermal fluctuations this difference is negligible and, subsequently, it is expected that distinct, welldefined microphase separated structures will not form in such systems. As both $\chi_{A S} N$ and $\chi_{B S} N$ increase, the free energy difference between the uniform phase and the non-uniform phases increases. This is most significant for the systems where the solvent is selective for the grafted block (Models 2 and 3). Some variation is also observed between the individual non-uniform phases, but generally the phase boundaries between these phases are not that sharp. 
Despite the overlapping of the free energies of the different phases in the low $\chi_{A S} N$ and $\chi_{B S} N$ phase diagrams, these simulations has proved useful in rationalizing the behavior of the various films. Indeed, as is discussed further below, the models with the lowest $\chi_{A S} N$ and $\chi_{B S} N$ values most accurately model the solvent washed films. Furthermore, the microphase separated structures formed after solvent annealing and the structures present in the meta-stable state of the thermally annealed films (generally hexagonal for the $\mathrm{dPS}_{47} \mathrm{PMMA}_{135} \mathrm{OH}$ films $\left(f_{P S}=0.24\right)$ and inverse hexagonal for the $\mathrm{dPS}_{62} \mathrm{PMMA}_{69} \mathrm{OH}\left(f_{P S}=0.45\right)$ films $)$, display features characteristic of both Models 1 and 2.

\section{Discussion}

The experimental systems most directly comparable with the SCFT simulations are the films that were washed with solvent and quickly dried (but not annealed). Specifically, the washed films may be compared with the results presented in Models 1, 4 and 5 as these models have $\chi_{A S}$ and $\chi_{B S}$ values that are closest to the solubility parameters calculated for the solvents used here (table $3 ; \chi N$ values of 70 and 100 give $\chi$ of $\sim 0.05$ and $\sim 0.07$ respectively). All of the solvent washed films generated the uniform phase, which is consistent with the small difference between the free energies of the uniform phase and the various non-uniform phases predicted by the simulations. This contrasts with non-grafted films, which frequently produce microphase separated structures when cast by spin-coating (a process equivalent to the rapid drying of grafted films). ${ }^{34}$

When the concentration of solvent incorporated into the films is decreased, as is the case with the solvent annealed films, microphase separation (albeit weak) to form non-uniform phases is observed. This is both in line with the dilution approximation and equivalent to increasing the magnitude of $\chi_{A S} N$ and $\chi_{B S} N$. The presence of some microphase separation in these films clearly indicates that there must be a meaningful difference in the free energies of the different phases (as is shown by Model 2), but the poorly defined phase boundaries observed in the AFM images (figure 2) points to significant incorporation of solvent into the region between the two polymer phases (figure 8). For the ethyl acetate 
washed system the phase behavior is most accurately described by Model 1. The other solvents show varying degrees of microphase separation suggesting greater solvent content and/or selectively for the non-grafted block, though, as is noted below, the latter is unlikely to be a significant factor.

For the thermally annealed films the film structure is dependent on the duration of the heating period. It is clear that the films undergo a two-step microphase separation process from an initially disordered state, through an ordered meta-stable state (displaying non-uniform structures), to a final equilibrium state. As noted above, the structure of the films prior to annealing is consistent with the results produced by the models with low $\chi_{A S} N$ and $\chi_{B S} N$ values, while in the meta-stable state the phase behavior is consistent with a model that is both selective for the grafted block and has higher $\chi_{A S} N$ and $\chi_{B S} N$ values.

The generation of the observed meta-stable state phase structures in the films washed with ethyl acetate, THF and DCM is in agreement with the calculated selectivity of these solvents (table 3 ). However, the same correlation with the toluene washed films runs contrary to the selectivity of the solvent and indicates that additional parameters need to be considered. Specifically, the interaction of the solvent with the substrate and the distribution of solvent in the film as a result of evaporation. In studying non-grafted PS-block-PMMA films, Perlich et al. observed an interaction between the solvent (toluene) and substrate (a silicon wafer), which lead to the formation of an enriched layer of solvent, 4 $\mathrm{nm}$ thick, at the film/substrate interface. ${ }^{24}$ For the films examined here an equivalent layer would effectively swell the grafted phase relative to the non-grafted block due to the inherent asymmetry of the film. Such an interaction was not explicitly included in the simulations reported here, but the net effect of this interaction is similar to the use of a solvent that is selective for the grafted block. It is also wellknown that evaporation of solvent from a film leads to a solvent gradient normal to the surface of the film as evaporation occurs only from the exposed surface of the film. ${ }^{24}$ Thus, even solvents that are selective for the non-grafted block may display an apparent selectivity for the grafted block.

In considering the similarities between the experimental and theoretical results it is apparent that the observed behavior can only be rationalized if the amount of solvent in the films in the meta-stable state 
is markedly less than that found in the films immersed in solvent or solvent annealed. Although this would seem self-evident, given the conditions to which the films are exposed during thermal annealing, we believe that this drives the formation of the different states. As has been shown in the literature, ${ }^{24}$ and supported by the results published here, cast polymer films contain a significant portion of solvent that is subsequently driven off during thermal annealing. It is also well-known that increased solvent content dramatically increases the polymer diffusion rate.

We propose therefore that prior to annealing the films are both in the uniform state (formed during washing with liquid solvent and preserved, in a kinetically trapped state, upon drying), and have a relatively high solvent content. Upon heating, the increased kinetic energy of the system leads to a fast reorganization of the polymer chains into a pseudo-equilibrium state that is defined by the fraction of solvent present. In this state, the meta-stable state, the residual solvent appears selective for the grafted block due to the combined effects of the evaporation gradient and the enrichment of the film with solvent at the film/substrate interface. If heating is stopped at this point the structure becomes frozen in due to the low polymer diffusion rate with low solvent contents and at low temperatures. However, additional heating will eventually drive off all of the residual solvent and the thermodynamic equilibrium of these films is then defined by the dry brush model. As noted earlier, the structure of the films in the dried brush state is dominated by the surface tension difference between the two polymer blocks. Consequently, both $\mathrm{dPS}_{47} \mathrm{PMMA}_{135} \mathrm{OH}$ and $\mathrm{dPS}_{62} \mathrm{PMMA}_{69} \mathrm{OH}$ are predicted to form the uniform phase.

The formation of meta-stable structures is not, however, reliant on the presence of a liquid solvent. When the films were treated with $\mathrm{scCO}_{2}$, a microphase separated meta-stable state was still observed. For these films, the only change that occurs during thermal annealing is a collapse of the void spaces introduced into the film by the $\mathrm{scCO}_{2}$ treatment. This volume change is equivalent to the volume change experienced by the solvent washed films as the solvent evaporates, and the presence of such a change goes some way to explaining the formation of the meta-stable state in these films. But the 
formation of phase separated structures in this films can only be rationalized by considering how the polymer chains rearrange in a dry film.

In the melt state, the motion of long, linear polymer chains is restricted by entanglements and, subsequently, diffusion of the chains occurs via reptation. For large polymers, chain diffusion is generally slow, with the diffusion rate being dependent on the temperature and inversely dependent on the square of the molecular weight. With grafted films, the situation is further complicated by the attachment of one end of the polymer chain to an immovable surface. This reduces the degrees of freedom available to the polymer chain and inhibits reptation. For grafted films it is therefore expected that the diffusion rate of the free end of the polymer chain will be higher than diffusion rate of the bound end of the polymer chain - leading to an asymmetry in the rate of reorganization.

For films treated with $\mathrm{scCO}_{2}$ this effect will manifest itself primarily as a faster collapse of the film at the air/film interface. With homopolymer brushes such an asymmetric collapse would not be particularly significant, however, with DCBs the effect is coincidental with the inherent asymmetry of the film - the reorganization of the film will therefore be faster for the non-grafted block, leading to faster collapse of that block and the apparent increase in the volume fraction of the grafted block. As with the solvent washed films, this relative expansion of the grafted block may effectively be modeled as the incorporation of a selective solvent into the film. A schematic of the proposed evolution of the various films is presented in figure 9.

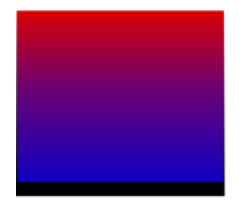

Washed Film

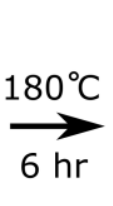

Meta-Stable State

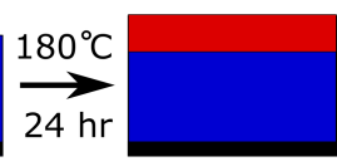

Equilibrium

State

Figure 9. Schematic of the structure evolution in grafted diblock films during thermal annealing.

In summary, it is proposed that the apparent asymmetry observed is the solvent washed films is due to enhanced swelling of the lower block caused by a strong solvent-substrate interaction and gradient in solvent concentration resulting from evaporation. With the $\mathrm{scCO}_{2}$ treated films the asymmetry arises 
from faster reorganization of the free-end of the polymer. However, it should be noted that both mechanisms may operates simultaneously. It is known that $\mathrm{scCO}_{2}$ interact attractively with silicon wafers and thus may cause additional expansion of the grafted block, ${ }^{40}$ while it is also conceivable that void spaces may be formed in the film washed with solvent. The latter case would arise if the rate of polymer diffusion decreased to such a point where it was no longer possible for the polymer chains to immediately compensate for solvent lost by evaporation. This will happen at some point during the evolution of the meta-stable state under annealing, but it will be difficult to conclude at what point one will become more dominant than the other.

\section{Conclusions}

In this work we have examined the behavior of a number of $\mathrm{dPS}_{\mathrm{x}} \mathrm{PMMA}_{\mathrm{y}} \mathrm{OH} \mathrm{DCBs}$ and observed dependencies on both the annealing time and nature of the solvent used to wash the films. Through the use of SCFT models we have been able to propose a mechanism that rationalizes the observed behavior in terms of contraction of the films during the annealing process; with the formation of the meta-stable state being most accurately described by the model for a DCB containing a solvent that is selective for

the lower block. This mechanism is not specific to the system examined here and should be applicable to other DCBs.

The behavior of the films following solvent annealing was also examined and it was observed that the films did not exhibit strong microphase separation, which is in agreement with both the SCFT models and the dilution approximation.

Acknowledgements The authors thank Prof Joe Keddie (University of Surrey) for the use of his ellipsometer, Martin Reeves and Dr John McKendrick (University of Reading) for assistance with the GCMS, and James Jennings and Prof Steve Howdle (University of Nottingham) for assistance with the $\mathrm{scCO}_{2}$ work. This research was supported by EPSRC grant EP/F029616/1. 
Supporting Information Available Topographic AFM images of the presented phase images are provided along with additional experimental results. This material is available free of charge via the Internet at http://pubs.acs.org.

\section{REFERENCES}

(1) Currie, E. P. K.; Norde, W.; Stuart, M. A. C. Adv. Colloid Interface Sci. 2003, 100, 205-65.

(2) Hamley, I. W. Prog. Polym. Sci. 2009, 34, 1161-210.

(3) Zhao, B.; Brittain, W. J. Prog. Polym. Sci. 2000, 25, 677-710.

(4) Edmondson, S.; Osborne, V. L.; Huck, W. T. S. Chem. Soc. Rev. 2004, 33, 14-22.

(5) O'Driscoll, B. M. D.; Oren, R.; Hamley, I. W. Polym. Adv. Tech. 2011, 22, 924-32.

(6) Ranjan, R.; Brittain, W. J. Macromolecules 2007, 40, 6217-23.

(7) Tchoul, M. N.; Fillery, S. P.; Koerner, H.; Drummy, L. F.; Oyerokun, F. T.; Mirau, P. A.; Durstock, M. F.; Vaia, R. A. Chem. Mat. 2010, 22, 1749-59.

(8) Matsen, M. W.; Bates, F. S. Macromolecules 1996, 29, 1091-8.

(9) Matsen, M. W.; Griffiths, G. H. Eur. Phys. J. E 2009, 29, 219-27.

(10) O'Driscoll, B. M. D.; Griffiths, G. H.; Matsen, M. W.; Perrier, S.; Ladmiral, V.; Hamley, I. W. Macromolecules 2010, 43, 8177-84.

(11) Phillip, W. A.; Hillmyer, M. A.; Cussler, E. L. Macromolecules 2010, 43, 7763-70.

(12) Yoshioka, A.; Tashiro, K. Polymer 2003, 44, 6681-8. 
(13) Keddie, J. L.; Jones, R. A. L. Isr. J. Chem. 1995, 35, 21-6.

(14) Forrest, J. A.; Dalnoki-Veress, K. Adv. Colloid Interface Sci. 2001, 94, 167-96.

(15) Naughton, J. R.; Matsen, M. W. Macromolecules 2002, 35, 5688-96.

(16) Hamley, I. W. The Physics of Block Copolymers; Oxford University Press: Oxford, 1998.

(17) Lodge, T. P.; Hanley, K. J.; Pudil, B.; Alahapperuma, V. Macromolecules 2003, 36, 816-22.

(18) Zhao, B.; Brittain, W. J. Macromolecules 2000, 33, 8813-20.

(19) Zhao, B.; Brittain, W. J.; Zhou, W.; Cheng, S. Z. D. J. Am. Chem. Soc. 2000, 122, 2407-8.

(20) Zhang, Y.; Gangwani, K. K.; Lemert, R. M. J. Supercrit. Fluids 1997, 11, 115-34.

(21) Arceo, A.; Green, P. F. J. Phys. Chem. B 2005, 109, 6958-62.

(22) Griffiths, G. H.; Vorselaars, B.; Matsen, M. W. Macromolecules 2011, DOI: $10.1021 / \mathrm{ma} 2003745$.

(23) Physical Properties of Polymers Handbook; 2nd ed.; Mark, J. E., Ed.; Springer: New York, NY, USA, 2007.

(24) Perlich, J.; Korstgens, V.; Metwalli, E.; Schulz, L.; Georgii, R.; Muller-Buschbaum, P. Macromolecules 2009, 42, 337-44.

(25) Garcia-Turiel, J.; Jerome, B. Colloid Polym. Sci. 2007, 285, 1617-23.

(26) Fitzgerald, T. G.; Farrell, R. A.; Petkov, N.; Bolger, C. T.; Shaw, M. T.; Charpin, J. P. F.; Gleeson, J. P.; Holmes, J. D.; Morris, M. A. Langmuir 2009, 25, 13551-60.

(27) Zhang, X. H.; Yager, K. G.; Kang, S. H.; Fredin, N. J.; Akgun, B.; Satija, S.; Douglas, J. F.; Karim, A.; Jones, R. L. Macromolecules 2010, 43, 1117-23. 
(28) Xuan, Y.; Peng, J.; Cui, L.; Wang, H.; Li, B.; Han, Y. Macromolecules 2004, 37, 7301-7.

(29) Chen, Y.; Huang, H.; Hu, Z.; He, T. Langmuir 2004, 20, 3805-8.

(30) O'Driscoll, B. M. D.; Newby, G. E.; Hamley, I. W. Polym. Chem. 2011, 2, 619-24.

(31) Wang, J.; Müller, M. Macromolecules 2009, 42, 2251-64.

(32) Guskova, O. A.; Seidel, C. Macromolecules 2011, 44, 671-82.

(33) Hansen, C. M. Hansen Solubility Parameters: A User's Handbook; 2nd ed.; CRC Press: Boca Raton, FL, USA, 2007.

(34) Kim, S.; Briber, R. M.; Karim, A.; Jones, R. L.; Kim, H. C. Macromolecules 2007, 40, 4102-5.

(35) Zhang, X. H.; Berry, B. C.; Yager, K. G.; Kim, S.; Jones, R. L.; Satija, S.; Pickel, D. L.; Douglas, J. F.; Karim, A. ACS Nano 2008, 2, 2331-41.

(36) Krausch, G.; Hipp, M.; Boeltau, M.; Marti, O.; Mlynek, J. Macromolecules 1995, 28, 260-3.

(37) Wang, D.; Fujinami, S.; Nakajima, K.; Nishi, T. Macromolecules 2010, 43, 3169-72.

(38) Tanaka, K.; Takahara, A.; Kajiyama, T. Macromolecules 1996, 29, 3232-9.

(39) Shim, J. J.; Johnston, K. P. Aiche J. 1989, 35, 1097-106.

(40) Sirard, S. M.; Ziegler, K. J.; Sanchez, I. C.; Green, P. F.; Johnston, K. P. Macromolecules 2002, $35,1928-35$. 
For Table of Contents Use Only

Structure Variation and Evolution in Microphase Separated Grafted Diblock Copolymer Films

Benjamin M.D. O’Driscoll, Guy H. Griffiths, Mark W. Matsen, and Ian W. Hamley

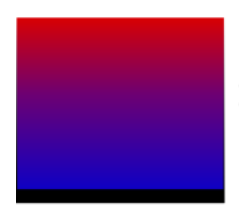

Washed

Film

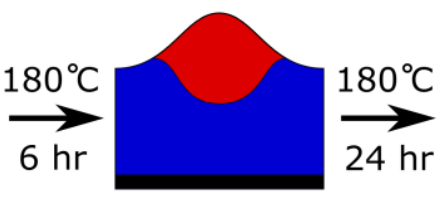

Meta-Stable State
Equilibrium

State 